\title{
The effect of failure criteria on risk-based inspection planning of offshore wind support structures
}

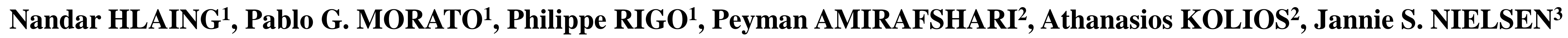

\section{Introduction}

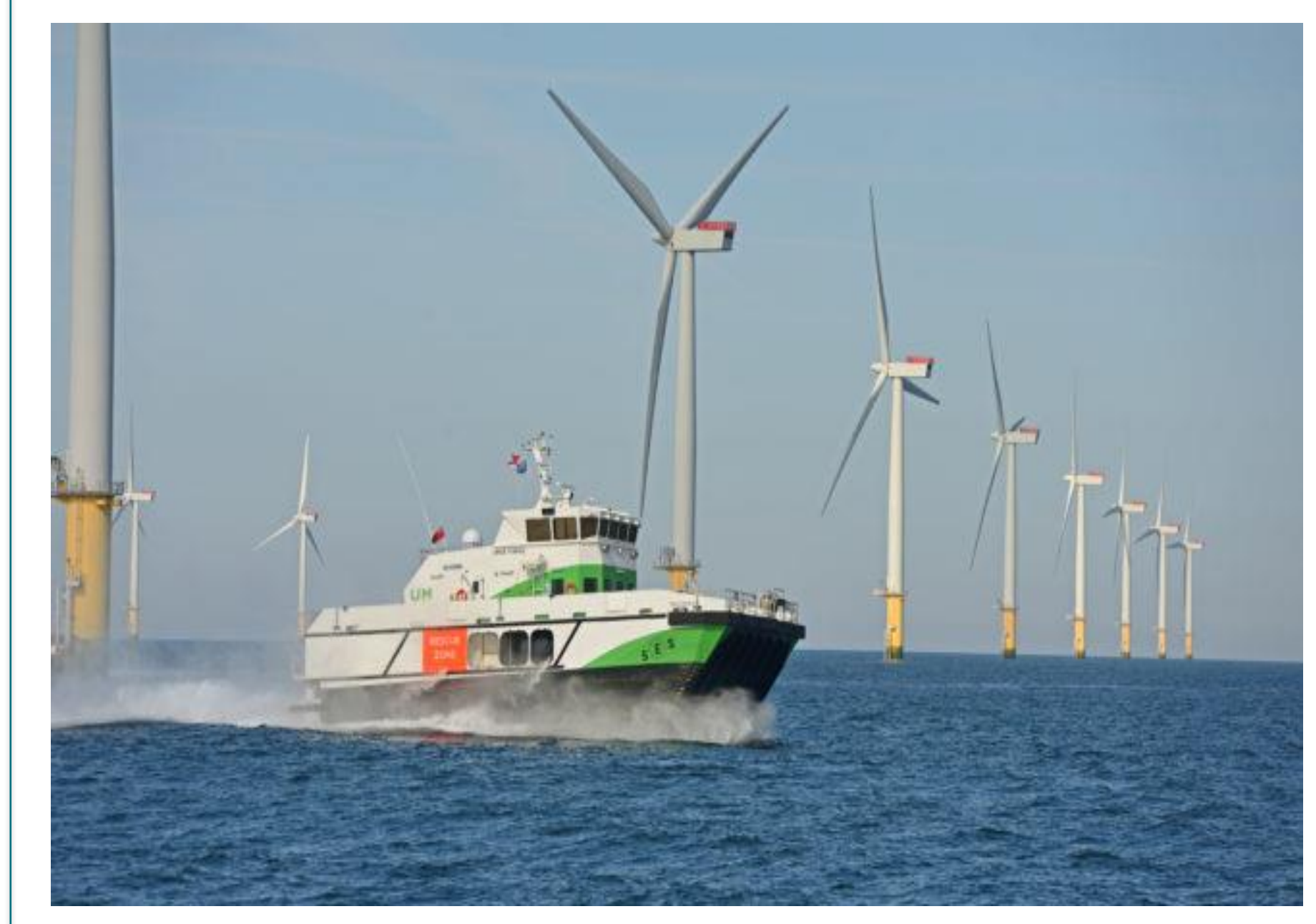

In risk-based inspection planning, the conventional through-thickness failure criterion is conservative for some redundant structures like jacket type OWTs. Thus, the use of a Failure Assessment Diagram (FAD) as a limit state function has been receiving increasingly attention. This work explores the influence of fracture mechanics models and failure functions on optimal inspection planning. The risk-based inspection framework is analysed with different FM models and failure functions for the case of a tubular joint and the effect of failure criteria on the optimal inspection plan is examined.

Research Aim: To identify the optimal inspection planning strategy

Impact: $\quad$ O\&M cost $(\approx 25 \%$ LCOE), Design optimization (Reduction of safety factor)

\section{Deterioration Modelling (Fatigue Model and Fracture Mechanics Model)}

Imperfections in the welding process can be considered as initial cracks and they grow under cyclic loading in a harsh environment. Paris-Erdogan's law is widely used in Linear Elastic Fracture Mechanics (LEFM) to model the crack growth

$$
\begin{aligned}
& \frac{d a}{d N}=C\left(\sqrt{\pi a} \Delta \sigma Y_{a}\right)^{m} \\
& \frac{d c}{d N}=C\left(\sqrt{\pi a} \Delta \sigma Y_{C}\right)^{m}
\end{aligned}
$$

Since the design of offshore substructures is based on fatigue model and the inspection planning demands for the crack growth, FM models are calibrated to the $\mathrm{SN}$ model. Initial crack size and crack growth parameters are calibrated for each FM model so that the reliability according to $\mathrm{SN}$ and FM approaches are similar over the lifetime.

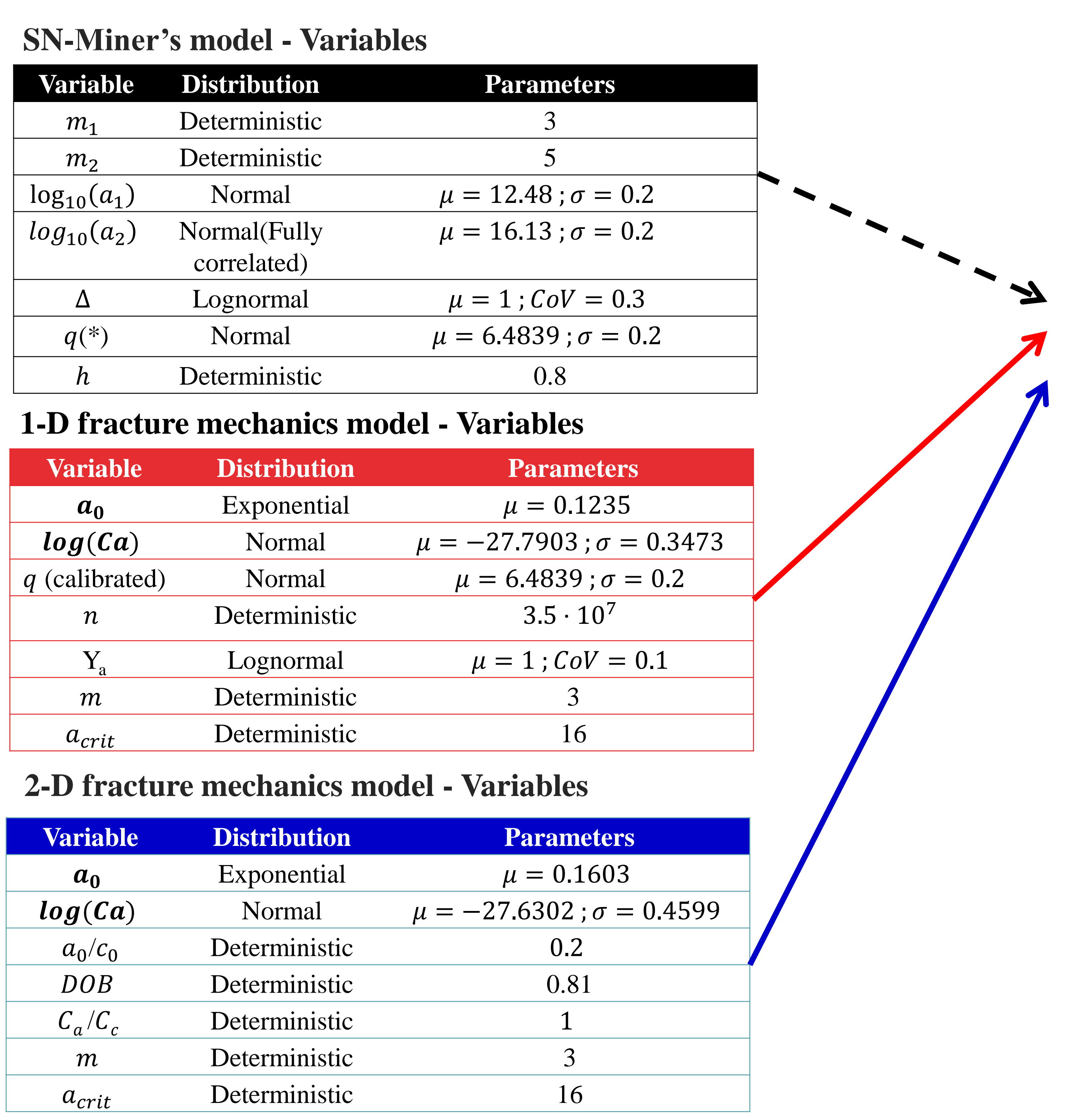

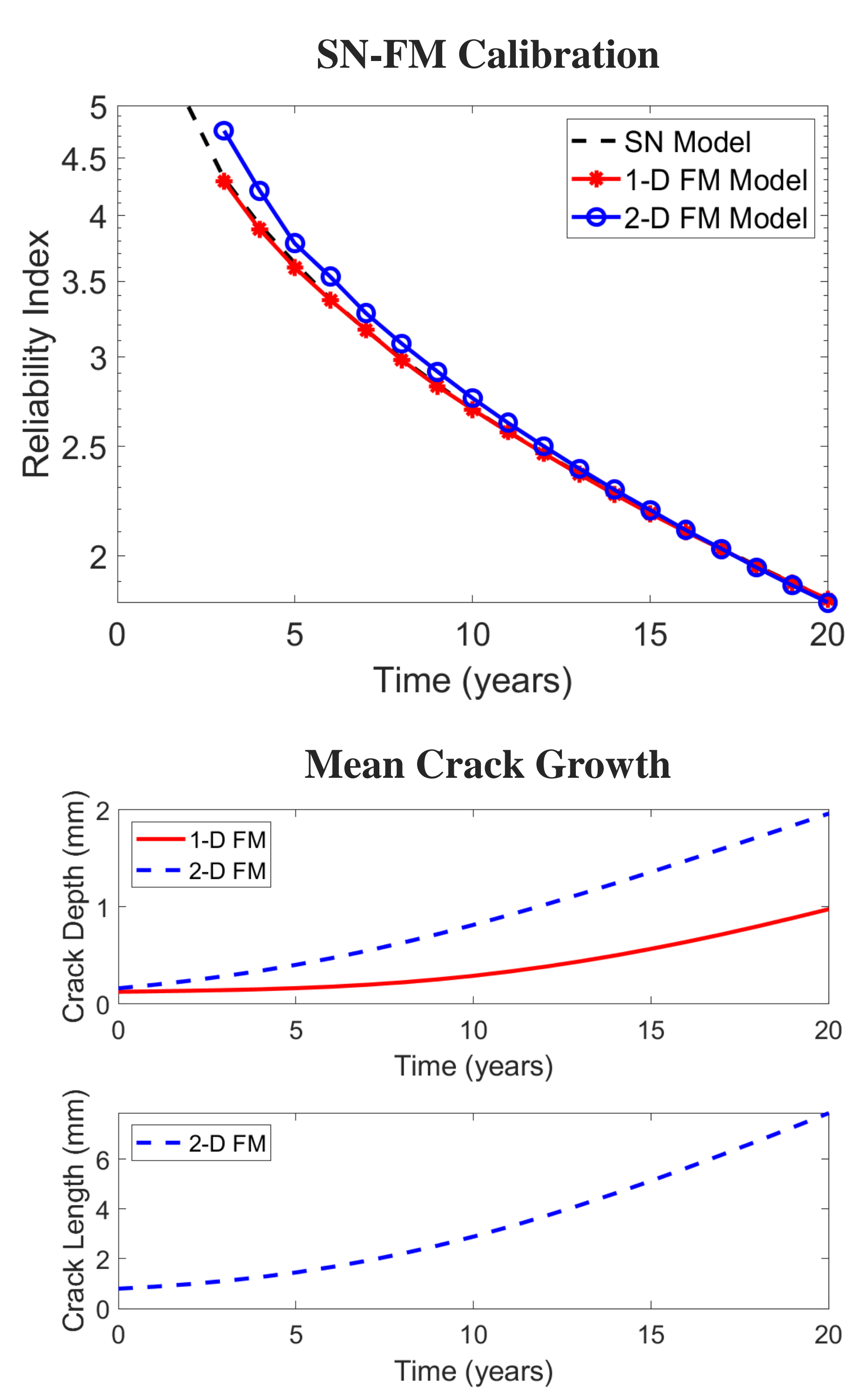

4. Results

Conventional through-thickness failure criterion $\longrightarrow$ Simplified FAD failure criterion

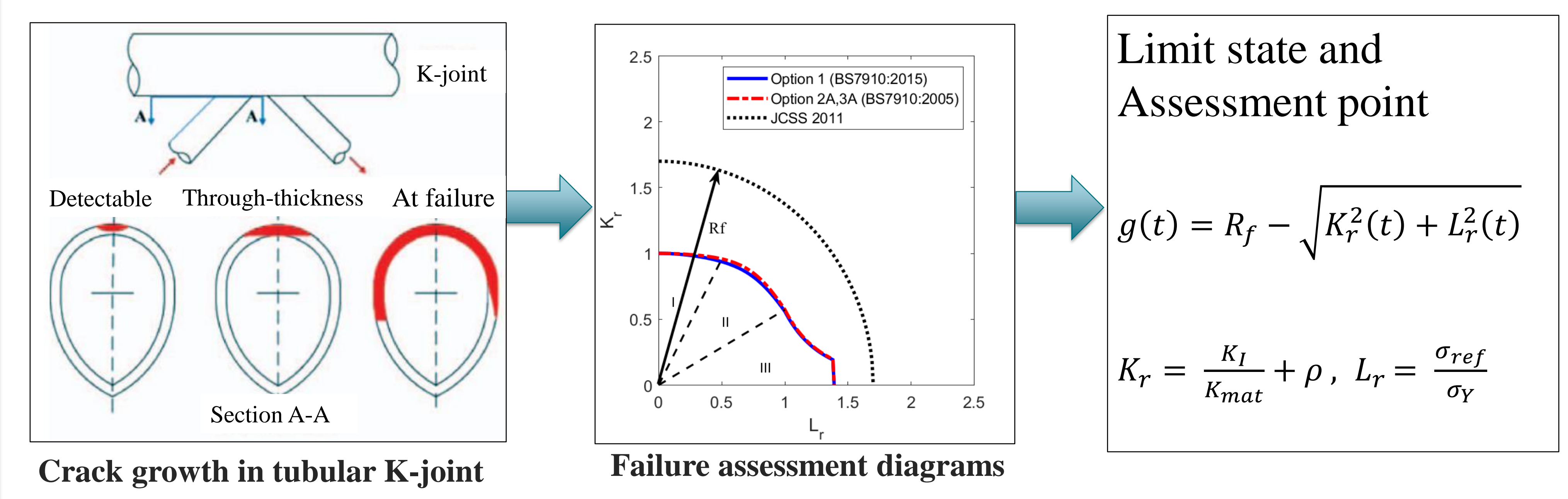

In 1-D FM + through thickness criterion, the optimal interval is 8 years and two inspections have to be carried out. The total cost is $\mathbf{7 0 5 7}$ money units. In 2-D FM + FAD criterion, the optimal plan is to inspect only once at year 11, resulting the total cost of $\mathbf{3 8 2 5}$ money units.

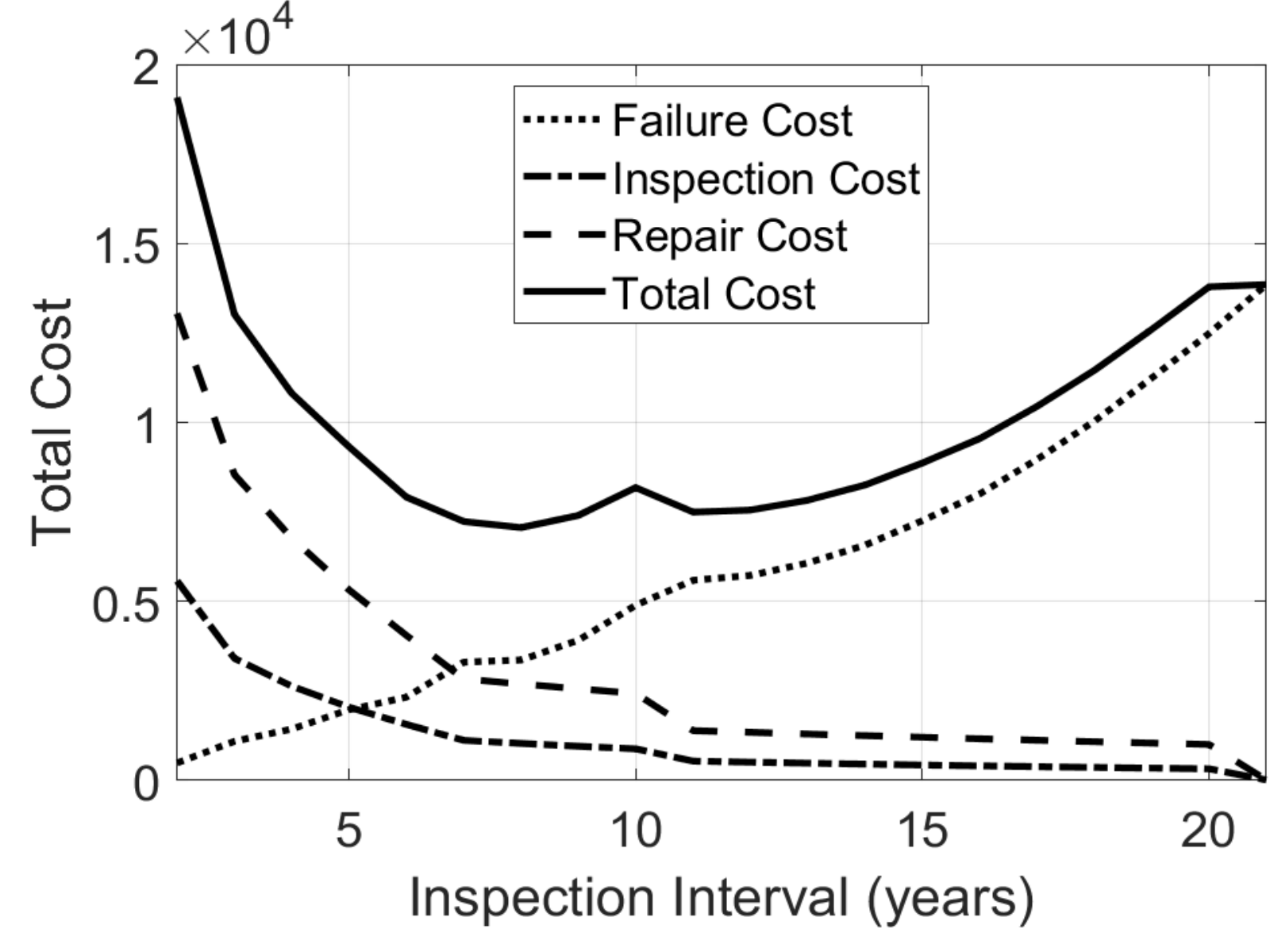

1-D FM Model + Through-thickness Failure

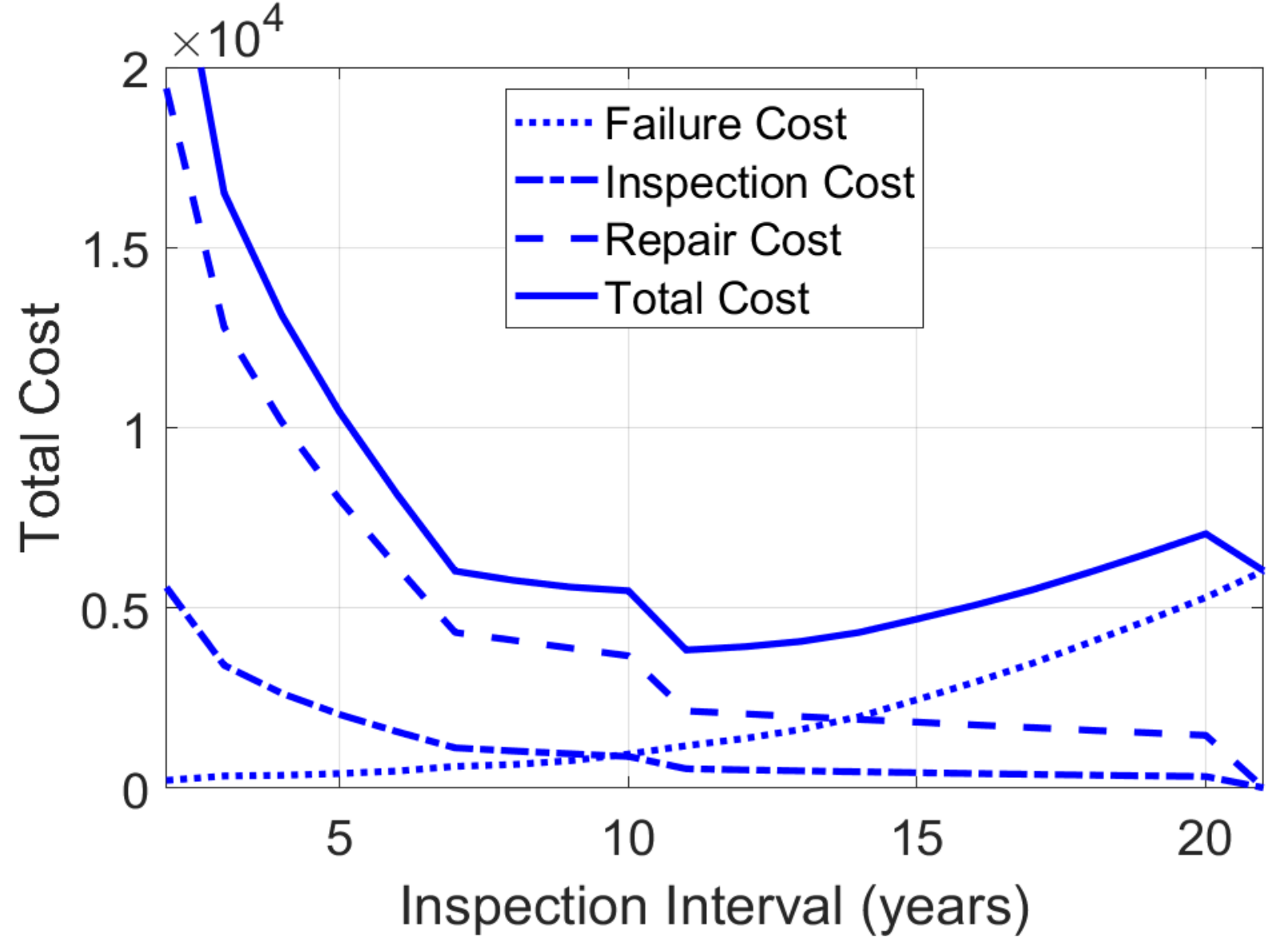

2-D FM Model + Simplified FAD Failure

The total expected cost is comprised of failure cost inspection cost and repair cost.

Failure cost: $10^{6}$ money units
Inspection cost: $10^{3}$ money units
Repair cost: $10^{4}$ money units
Discount rate: $6 \%$

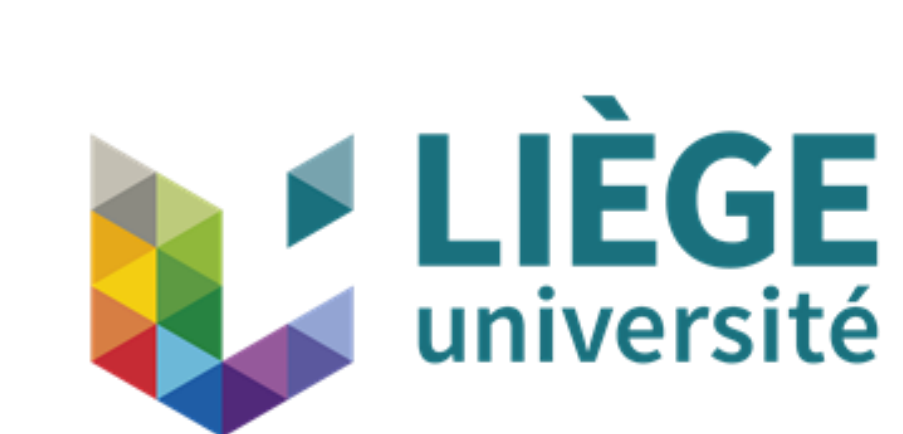

\section{$\pi$}

\section{Conclusion}

The choice of failure criteria and FM models affect the optimal inspection planning strategy. For redundant structures with high fracture toughness, using FAD failure criteria yields less total expected cost. The decision maker(s) should keep it in mind and the appropriate failure criterion should be wisely chosen. 